\title{
Role of host angiotensin II type 1 receptor in tumor angiogenesis and growth
}

\author{
Kimiyasu Egami, ${ }^{1,2}$ Toyoaki Murohara, ${ }^{1,3,4}$ Toshifumi Shimada, ${ }^{1,3}$ Ken-ichiro Sasaki, 1,3 \\ Satoshi Shintani, ${ }^{1,3}$ Takeshi Sugaya, ${ }^{5}$ Masahiro Ishii, ${ }^{1,2}$ Teiji Akagi, ${ }^{1,2}$ Hisao Ikeda, ${ }^{1,3}$ \\ Toyojiro Matsuishi, ${ }^{1,2}$ and Tsutomu Imaizumi ${ }^{1,3}$
}

\author{
${ }^{1}$ The Cardiovascular Research Institute, \\ ${ }^{2}$ Department of Pediatrics, and \\ ${ }^{3}$ Department of Internal Medicine III, Kurume University School of Medicine, Kurume, Japan \\ ${ }^{4}$ Department of Cardiology, Nagoya University Graduate School of Medicine, Nagoya, Japan \\ ${ }^{5}$ Discovery Research Laboratory, Tanabe Seiyaku Co., Osaka, Japan
}

\begin{abstract}
Although the renin angiotensin system (RAS) is a major regulator of vascular homeostasis, the role of the RAS in tumor angiogenesis is little understood. Here we show that host angiotensin II (ATII) type 1 (AT1) receptor plays an important role in angiogenesis and growth of tumor cells engrafted in mice. Subcutaneous B16-F1 melanoma-induced angiogenesis as assessed by tissue capillary density and microangiography was prominent in WT mice but was reduced in AT1a receptor-deficient $\left(A T 1 a^{-/-}\right)$ mice. Consequently, tumor growth rate was significantly slower, and the mouse survival rate was greater, in $A T 1 a^{-/-}$mice than in WT mice. Tumor growth was also reduced in WT mice treated with TCV-116, a selective blocker of AT1 receptor. Because the $\beta$-galactosidase gene was inserted into the AT1a gene locus in $A T 1 a^{-/-}$mice, the site of $\beta$-galactosidase expression represents the AT1a receptor expression in these mutant mice. In tumor-implanted $A T 1 a^{-/-}$mice, the major site of the $\beta$-galactosidase expression was macrophages in tissues surrounding tumors. Moreover, the number of infiltrated macrophages was significantly lower in $A T 1 a^{-/-}$mice than in WT mice, and double-immunofluorescence staining revealed that these macrophages expressed VEGF protein intensively. Therefore, the host ATII-AT1 receptor pathway supports tumor-associated macrophage infiltration, which results in enhanced tissue VEGF protein levels. The host ATII-AT1 receptor pathway thereby plays important roles in tumor-related angiogenesis and growth in vivo.
\end{abstract}

J. Clin. Invest. 112:67-75 (2003). doi:10.1172/JCI200316645.

\section{Introduction}

The renin-angiotensin system (RAS) plays important roles in the regulation of vascular homeostasis (1). A recent large-scale clinical trial for hypertension demonstrated that angiotensin-converting enzyme (ACE) inhibitors reduced not only the mortality rate due to cardiovascular diseases but also the rate due to malignant tumors (2). Because tumor growth depends on angiogenesis $(3,4)$, one may speculate that ACE

Received for publication August 12, 2002, and accepted in revised form April 29, 2003.

Address correspondence to: Toyoaki Murohara, Department of Cardiology, Nagoya University Graduate School of Medicine, 65 Tsurumai, Showa-ku, Nagoya 466-8550, Japan.

Phone: 81-52-744-2149; Fax: 81-52-744-2157;

E-mail: murohara@med.nagoya-u.ac.jp.

This work was presented in part at the Annual Scientific Sessions of the American Heart Association in Chicago, Illinois, USA, on November 17, 2002.

Conflict of interest: The authors have declared that no conflict of interest exists.

Nonstandard abbreviations used: renin-angiotensin system (RAS); angiotensin-converting enzyme (ACE); angiotensin II (ATII); ATII type 1 (AT1); AT1a receptor-deficient $\left(A T 1 a^{-/-}\right)$; $A T 1$ a receptor-deficient heterozygous $\left(A T 1 a^{+/-}\right)$;

3, 3'-diaminobenzidine (DAB); tumor-associated macrophage (TAM); phycoerythrin (PE); monocyte chemoattractant protein-1 (MCP-1); O-(chloroacetyl-carbamoyl)fumagillol (TNP-470). inhibitors might have reduced tumor angiogenesis and growth. In fact, an ACE inhibitor, captopril, has been shown to inhibit tumor angiogenesis (5). In other experimental models, however, for example in a reparative hindlimb ischemia model $(6,7)$, ACE inhibitors augmented angiogenesis, leaving the role of the RAS in angiogenesis unclear.

In many previous studies, ACE inhibitors were mainly used to suppress the functions of the RAS as a pharmacological tool; however, ACE inhibitors suppress not only the synthesis of angiotensin II (ATII) but also the activity of kininase II (8). Consequently, ACE inhibitors increase tissue bradykinin concentration, which stimulates endothelial NO release and thereby affects angiogenesis $(8,9)$. Moreover, ATII is synthesized by another enzyme, chymase (10). Therefore, the use of ACE inhibitors alone cannot fully elucidate the precise role of ATII in angiogenesis in vivo.

To further elucidate the role of ATII in tumor-related angiogenesis, we sought to determine the effects of the blockade of functional ATII receptor on angiogenesis in vivo. There are two major subtypes of ATII receptors, AT type 1 and 2 (AT1 and AT2) (11). The AT1 receptor is further subdivided into AT1a and AT1b in murine species. Most of the ATII functions in the cardiovascular system are mediated through the AT1 receptor, and 
in rodents they are mediated through the AT1a receptor (11-14). We took advantage of employing genetically modified AT1a receptor-deficient $\left(A T 1 a^{--}\right)$mice, which we generated previously (15), and examined the role of the ATII-AT1a receptor system in host animals in tumor growth and angiogenesis in vivo. We also examined the effects of a selective AT1 receptor antagonist TCV-116 on tumor angiogenesis and growth in WT animals (12).

\section{Methods}

AT1 a receptor-deficient mouse. To obtain AT1a receptor-deficient heterozygous $\left(A T 1 a^{+/-}\right)$mice, a germline chimera derived from TT2 ES cells with a targeted mutation of the AT1a gene (15), was backcrossed for five generations with C57BL/6 mice (16). The resulting $A T 1 a^{+/-} \mathrm{F}_{5}$ mice were intercrossed to generate the homozygous $\left(A T 1 a^{-/-}\right)$mutant mice. In the $A T 1 a^{-/-}$ mice the AT1a gene was deleted, and the $\beta$-galactosidase gene was inserted into its original gene locus. Consequently, $A T 1 a$ receptor expression can be analyzed by the $\beta$-galactosidase gene or protein expression in $A T 1 a^{-/-}$ mice $(15,16)$. As WT (or $\left.A T 1 a^{+/+}\right)$control mice, C57BL/6 mice were obtained from CLEA Japan Inc. (Tokyo, Japan). Male mice, 8-10 weeks old, were used.

Tumor implantation model. Study protocols were approved by the Institutional Animal Care and Use Committee of Kurume University School of Medicine. We used a mouse model of tumor angiogenesis as described previously (17). We employed two different cell lines, B16-F1 melanoma and QRsP-11 fibrosarcoma cells, which can grow in the C57BL/6 strain mouse $(17,18)$. B16-F1 melanoma cells (batch no. 1224725 , flask passage no. 26) were purchased from the American Type Culture Collection (Manassas, Virginia, USA). Cells were cultured in DMEM supplemented with 4 mM L-glutamine, $4.5 \mathrm{~g} / 1$ glucose, $10 \% \mathrm{FBS}$, and antibiotics (Life Technologies Inc., Gaithersburg, Maryland, USA) under humidified air with $5 \% \mathrm{CO}_{2}$ at $37^{\circ} \mathrm{C}$. Before use, the melanoma cell monolayer in culture was washed and detached with PBS containing 0.25\% trypsin and $0.03 \%$ EDTA and then pelleted by brief centrifugation at $100 \mathrm{~g}$. The supernatant was removed, cell pellets were resuspended in PBS, and the cell number was counted (Sysmex Inc., Tokyo, Japan). The cell concentration was finally adjusted to $5 \times 10^{6} \mathrm{cells} / \mathrm{ml}$, and $200 \mu \mathrm{l}$ of the suspension was injected subcutaneously into WT mice $(n=11)$ and $A T 1 a^{-/-}$mice $(n=12)$ in the dorsal skin using a disposable tuberculin syringe and a 27 -gauge needle under anesthesia $(30 \mathrm{mg} / \mathrm{kg}$ pentobarbital, intraperitoneally). QRsP-11 fibrosarcoma cells were kindly provided by F. Okada at Hokkaido University, Sapporo, Japan. Cells were cultured in MEM supplemented with $110 \mathrm{mg} / \mathrm{l}$ sodium pyruvate, nonessential amino acids, L-glutamine $(292.3 \mathrm{mg} / \mathrm{l}), 10 \% \mathrm{FBS}$, and antibiotics under humidified air with $5 \% \mathrm{CO}_{2}$ at $37^{\circ} \mathrm{C}$. Cells were otherwise identically isolated from culture dishes, as were B16-F1 melanoma cells. The cell concentration was finally adjusted to $2 \times 10^{6}$ cells $/ \mathrm{ml}$, and $200 \mu \mathrm{l}$ of the suspension was injected subcutaneously into WT mice $(n=22)$ and $A T 1 a^{-/-}$mice $(n=15)$ in the dorsal skin.

At several time points after tumor implantation, the size of tumor was measured using a caliper. The tumor volume was calculated according to the formula $\left(V=\left[L \times W^{2}\right] \times 0.52\right)$, where $V=$ volume, $L=$ length, and $W=$ width (length is greater than width) (19). Before cell implantation, systemic arterial blood pressure and heart rate were measured in the conscious state using a small animal tail-cuff BP analyzer (MK2000; Muromachi Kikai Co. Ltd., Tokyo, Japan).

Microangiography. Postmortem tumor microangiography was performed on day 21 after melanoma cell implantation in both WT and $A T 1 a^{-/-}$mice $(n=4$ in each group). Under deep anesthesia with pentobarbital sodium $(60 \mathrm{mg} / \mathrm{kg}$, intraperitoneally), the thoracic cavity was rapidly opened, and a 24-gauge soft-tip catheter was inserted through the apex into the left ventricular cavity. The aorta was gently perfused with $1 \mathrm{ml}$ of warm saline $\left(37^{\circ} \mathrm{C}\right)$ containing heparin $(10 \mathrm{U} / \mathrm{ml})$, which was followed by injection of filtered barium sulfate $(0.25$ g/ml, $0.3 \mathrm{ml}$; Fushimi Corp., Kagawa, Japan). The whole bodies of the mice were immediately fixed with $20 \%$ formalin solution overnight, and a portion of primary tumor and adjacent subcutaneous tissues surrounding the tumors was isolated as a tissue block. Multiple tissue slices $1 \mathrm{~mm}$ thick were prepared by using a microtome through their center, taking surrounding tissue. The slices from each tumor were subjected to microangiography using an $\mathrm{x}$-ray mammography system (Senographe 500T; GE Medical Systems-Europe, Paris, France) (20).

Capillary-density analysis. On day 21 after melanoma implantation, tumors and subcutaneous tissues surrounding tumors (approximately $3 \mathrm{~mm}$ from the tumor margin) were carefully isolated, fixed in methanol overnight, and embedded in paraffin. Multiple tissue slices $5 \mu \mathrm{m}$ thick were prepared from both WT and $A T 1 a^{-/-}$mice ( $n=6$ in each group). Endothelial cells were immunohistochemically stained to examine the capillary density. In brief, capillary endothelium was identified by staining with either a rat anti-mouse CD31 mAb (PharMingen, San Diego, California, USA) or a rat antihuman vWF mAb (DAKO A/S, Glostrup, Denmark), followed by immunoperoxidase staining using a commercially available kit (VectaStain ABC-PO; Vector Laboratories, Burlingame, California, USA). The rat antihuman $\mathrm{VWF}$ mAb cross-reacts with mouse endothelial cells. Final color products were developed using a solution containing 3, $3^{\prime}$-diaminobenzidine (DAB) and $\mathrm{NiCl}_{2}$ (DAB substrate kit; Vector Laboratories).

For the analysis of the capillary density in skeletal muscles on day 21, tissues located just below tumors were isolated and snap-frozen in OCT compound with liquid $\mathrm{N}_{2}$. Five-micrometer-thick frozen sections were prepared from each specimen so that the muscle fibers were oriented in a transverse fashion. The sections were stained for alkaline phosphatase to detect capillary 
endothelial cells within skeletal muscle tissues as described previously (21). Fifteen random microscopic fields from three different sections in each tissue were examined for the presence of capillary endothelial cells under light microscopy, and capillary density was expressed as the number of capillaries per high-power field $(\times 400)$. The final capillary-density score represents an average of all fields.

Histological analysis of tumor-associated macrophage infiltration. Macrophages express AT1a receptors (22), and ATII has been shown to evoke inflammatory responses in various tissues $(16,23)$. Moreover, macrophage infiltration is an important promoter for tumor angiogenesis and growth (24-27), and these cells are called tumor-associated macrophages (TAMs). We thus examined TAM infiltration around melanoma tissues and compared the number of infiltrated TAMs between WT and $A T 1 a^{-/-}$mice. Leukocyte infiltration was first analyzed by a standard $H \& E$ staining method in multiple sections prepared from paraffin-embedded tissues. Cryostat sections $5 \mu \mathrm{m}$ thick from tumors and surrounding subcutaneous tissues were then mounted on silicone-coated slides, and TAMs were immunohistochemically identified using a rat anti-mouse macrophage mAb (clone F4/80; Serotec Ltd., Oxford, United Kingdom) followed by an immunoperoxidase staining using the VectaStain ABC-PO kit and DAB substrate (Vector Laboratories). TAMs were defined as infiltrated macrophages located around tumors and within a distance of 3,000 $\mu \mathrm{m}$ from the tumor margin. Histological analysis of VEGF expression. Infiltrated macrophages release angiogenic cytokines, including VEGF, which promote angiogenesis (23). We thus examined VEGF protein expression in TAMs by a double-immunofluorescence staining. Cryostat sections 5 $\mu \mathrm{m}$ thick from tumors and surrounding subcutaneous tissues were mounted on silicone-coated slides. They were incubated overnight at $4{ }^{\circ} \mathrm{C}$ with an anti-mouse VEGF mAb (Santa Cruz Biotechnology, Santa Cruz, California, USA) and with an anti-mouse macrophage $\mathrm{mAb}(\mathrm{F} 4 / 80)$ in a moist chamber. The slides were then incubated for 30 minutes at $37^{\circ} \mathrm{C}$ with a FITC-conjugated anti-goat IgG secondary $\mathrm{Ab}$ (Zymed Laboratories Inc., San Francisco, California, USA) to detect VEGF. They were then further incubated for 30 minutes at $37^{\circ} \mathrm{C}$ with a PE-conjugated anti-rat IgG (Serotec Ltd.) to detect macrophages. The slides were examined and photographed under fluorescence microscopy (DIAPHOT 300; Nikon Corp., Tokyo, Japan).

$R T-P C R$. We examined AT1a receptor mRNA expression by RT-PCR in isolated tissues. To determine whether differential localization of the AT1a receptor expression occurs in and around tumors, we isolated tumor tissues, subcutaneous tissues surrounding tumors, and tails from WT and $A T 1 a^{-/-}$mice on day 21 . Total RNA was extracted from each tissue using a guanidium thiocyanate-phenol chloroform solution (TRIzol reagent; Life Technologies Inc.), quantified by measuring absorption at $260 \mathrm{~nm}$ and subjected to
RT-PCR. Total RNA was reverse-transcribed using random hexamer primers and $\mathrm{RNase} \mathrm{H}^{-}$reverse transcriptase (Superscript II; Life Technologies Inc.) with $2 \mu \mathrm{g}$ of total RNA per sample. AT1a receptor mRNA in isolated tissues and in cultured B16-F1 melanoma cells was determined using primers $5^{\prime}$-TCACCTGCATCATCATCTGG-3' (sense) and 5'-AGCTGGTAAGAATGATTAGG-3' (antisense), which yielded a 201-bp product.

In $A T 1 a^{-/}$mice, the $A T 1 a$ receptor gene was deleted, and the $\beta$-galactosidase gene was inserted into its locus. Consequently, AT1a receptor expression was represented by $\beta$-galactosidase in $A T 1 a^{-/-}$mice (15). We therefore performed RT-PCR analysis for detecting the $\beta$-galactosidase mRNA in isolated $A T 1 a^{-/-}$mice-derived tissues. For this purpose, a set of primers, $5^{\prime}$-TCAGATGTGCGGCGAGTTGCGT-3' (sense) and 5'-CAGAGGATGATGCTCGTGACGG-3' (antisense), was used, which yielded a 452-bp product. Primers specific for mouse GAPDH were used as a control RT-PCR.

Histological evaluation of the expression of $\beta$-galactosidase. Cryostat sections $5 \mu \mathrm{m}$ thick from tumors and surrounding subcutaneous tissues were mounted on silicone-coated slides and briefly dried. Slides were incubated overnight at $4^{\circ} \mathrm{C}$ with an anti- $\beta$-galactosidase $\mathrm{mAb}$ (HyTest Ltd., Turku, Finland) and with an antimouse macrophage $\mathrm{mAb}(\mathrm{F} 4 / 80)$ in a moist chamber. The slides were then incubated for 30 minutes at $37^{\circ} \mathrm{C}$ with a FITC-conjugated anti-mouse IgG secondary Ab (Rockland Immunochemicals Inc., Gilbertsville, Pennsylvania, USA) to detect $\beta$-galactosidase. They were further incubated for 30 minutes at $37^{\circ} \mathrm{C}$ with a PE-conjugated anti-rat IgG (Serotec Ltd.) to detect macrophages. The slides were examined under fluorescence microscopy (DIAPHOT 300; Nikon Corp.).

Measurement of tissue monocyte chemoattractant protein-1 and VEGF levels. Because infiltration of macrophages is associated with expression of chemokine MCP-1, we determined tissue levels of monocyte chemoattractant protein-1 (MCP-1) protein using ELISA. Subcutaneous tissues surrounding tumors $3 \mathrm{~mm}$ thick were isolated from the surface of tumors, and tissues were homogenized and centrifuged for 15 minutes at $3,500 \mathrm{~g}$ at $4^{\circ} \mathrm{C}$. Supernatant was then recovered, and MCP- 1 levels were determined using a mouse MCP-1 ELISA kit (Quantikine M; R\&D Systems Inc., Minneapolis, Minnesota, USA). Because infiltrated macrophages release an angiogenic cytokine VEGF, we also determined the tissue VEGF levels using a mouse VEGF ELISA kit (Quantikine $\mathrm{M} ; \mathrm{R} \& D$ Systems Inc.). Finally, VEGF protein levels within tumor masses without necrosis were also determined using the ELISA method. Data are expressed as picograms per milligram of tissue.

Effects of an angiogenesis inhibitor O-(chloroacetyl-carbamoyl)fumagillol on tumor angiogenesis and growth in WT and $A T 1 a^{-/}$mice. We examined whether angiogenesis inhibitor $O$-(chloroacetyl-carbamoyl)fumagillol (TNP-470) $(28,29)$, could inhibit melanoma growth and angiogenesis in vivo. TNP-470 treatment was initiated on the day of tumor implantation, and mice received TNP-470 
$(30 \mathrm{mg} / \mathrm{kg}$, subcutaneously) every other day. This treatment regimen and the dose of TNP-470 have been shown to effectively block angiogenic response in murine experimental models (29).

Effects of a selective AT1 receptor blocker TCV-116 on tumor angiogenesis and growth in WT mice. We evaluated whether pharmacological blockade of the AT1 receptor function by treatment with TCV-116, a potent and selective AT1 receptor blocker $(12,30,31)$, could inhibit melanoma growth and angiogenesis in WT mice in vivo. Some mice received TCV-116 treatment $(10 \mathrm{mg} / \mathrm{kg} /$ day, orally $)$ initiated 7 days prior to tumor implantation, and the tumor growth was compared between TCV-116-treated $(n=17)$ mice and untreated WT mice $(n=16)$.

Statistics. All values are presented as mean plus or minus SE. Data were subjected to paired or unpaired Student $t$ tests for comparison between WT and $A T 1 a^{-/-}$mice. When comparing more than three groups, ANOVA with post hoc analysis was used. The rate of mouse survival was compared between the tumor-implanted WT and $A T 1 a^{-/-}$groups by the Kaplan-Meier method (32). $P$ values of less than 0.05 were considered to be statistically significant.

\section{Results}

Tumor growth in WT mice and the effects of TNP-470. First, to evaluate whether subcutaneous melanoma growth depends on angiogenesis, B16-F1 melanoma cells $\left(10^{6} /\right.$ animal $)$ were implanted into the dorsal skin tissues of either WT mice or $A T 1 a^{-/-}$mice, and we examined the effects of TNP-470, a potent angiogenesis inhibitor, on tumor growth. The growth of engrafted tumors was significantly inhibited in both WT mice and $A T 1 a^{-/-}$mice receiving TNP-470 compared with control WT and $A T 1 a^{-/-}$mice (Figure 1, a and b). The inhibitory efficacy of TNP-470 on tumor growth was more prominent in WT mice compared with $A T 1 a^{-1-}$ mice. Postmortem tumor microangiography on day 21 revealed that the formation of visible tumor-associated vessels visible with microangiography was less potent in tumors engrafted in mice receiving TNP-470 in both WT mice and $A T 1 a^{-/-}$mice, compared with those engrafted in mice receiving saline (Figure 1c). These data suggest that subcutaneous melanoma growth is indeed dependent on angiogenesis.

Tumor growth and mouse survival in WT and $A T 1 a^{-/-}$ mice. B16-F1 melanoma cells $\left(10^{6}\right.$ cells/animal) were implanted into the dorsal skin of WT and $A T 1 a^{-/-}$ mice. The two groups of mice exhibited similar tumor engraftment rates during the first 7 days after implantation; however, tumors engrafted in $A T 1 a^{-/-}$mice continued to grow more slowly than did tumors in WT mice. By postimplantation day 21, the mean size of tumors grafted in $A T 1 a^{-/-}$mice was significantly smaller than that in WT mice (Figure 2a). The KaplanMeier analysis showed that the rate of host mouse survival was significantly higher in the $A T 1 a^{-/-}$group than in the WT group (Figure $2 b$ ), consistent with the data of tumor growth.
QRsP-11 fibrosarcoma cells $\left(4 \times 10^{5}\right.$ cells/animal) were implanted into the dorsal skin of WT and $A T 1 a^{-/-}$mice. The two groups of mice exhibited similar tumor engraftment rates during the first 7 days. Tumors engrafted in $A T 1 a^{-/-}$mice grew more slowly than did tumors in WT mice, however. By postimplantation day 28 , the mean size of tumors grafted in $A T 1 a^{-/-}$mice was significantly smaller than that in WT mice (Figure 2c). The Kaplan-Meier analysis showed that the rate of host mouse survival was significantly higher in the $A T 1 a^{-/}$group than in the WT group up to day 42 (Figure $2 d$ ), consistent with the data of tumor growth. These data suggest an important role of the host AT1a receptor in supporting tumor growth. a
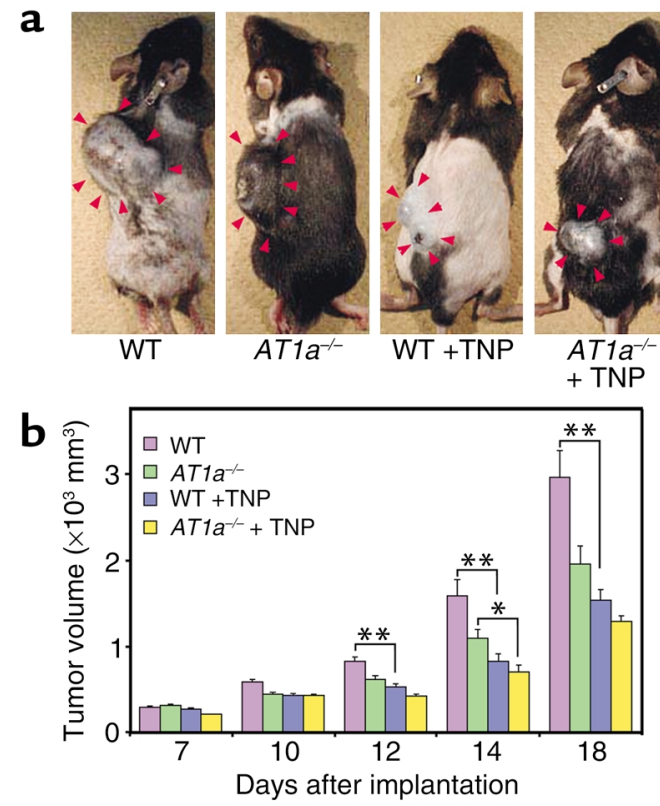

c

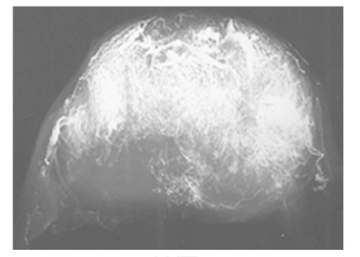

WT

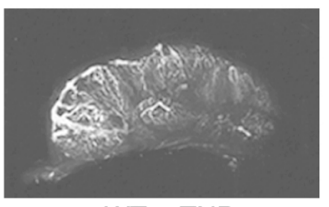

WT + TNP

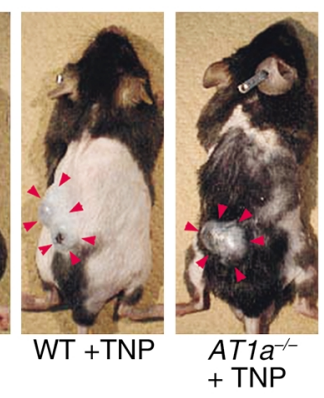

*

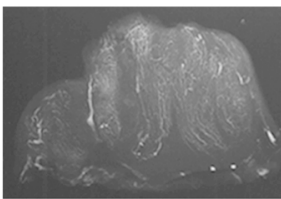

$\operatorname{ATTa}^{-/-}$

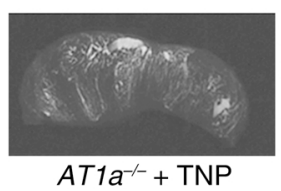

$A T 1 a^{-/}+$TNP

\section{Figure 1}

Angiogenesis inhibitor TNP-470 suppresses tumor angiogenesis and growth. (a and $\mathbf{b}$ ) A total of $10^{6} \mathrm{~B} 16-\mathrm{F} 1$ melanoma cells were implanted subcutaneously into WT $(n=37)$ and $A T 1 a^{-/-}$mice $(n=33)$ with or without TNP-470 administration. TNP-470 administration significantly inhibited tumor growth in both WT mice $(n=20)$ and AT1 $a^{-/-}$mice $(n=17)$. The inhibitory efficacy of TNP-470 was prominent in WT mice as compared with $A T 1 a^{-/-}$mice. ${ }^{*} P<0.05$; ${ }^{*} P<0.01$. (c) Representative $x$-ray microangiograms of melanomas grown in WT and $A T 1 a^{-1-}$ mice with or without TNP-470. Administration of TNP-470 reduced angiographically visible tumor-related angiogenesis. TNP, TNP-470. 


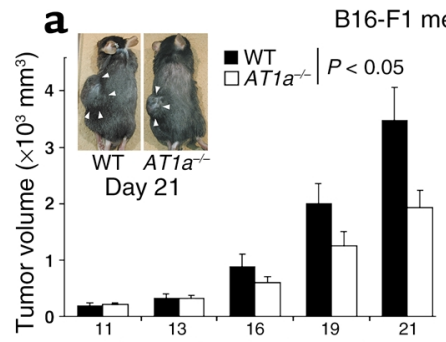

Days after implantation

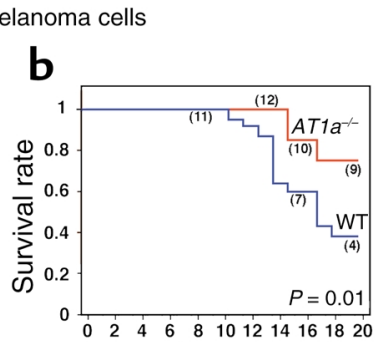

Days after implantation

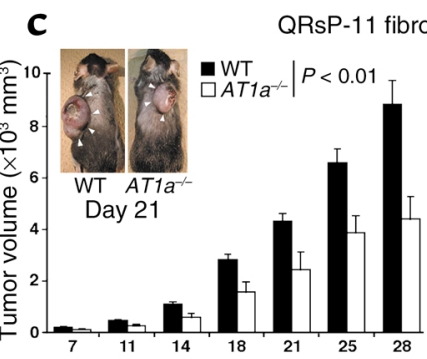

Days after implantation

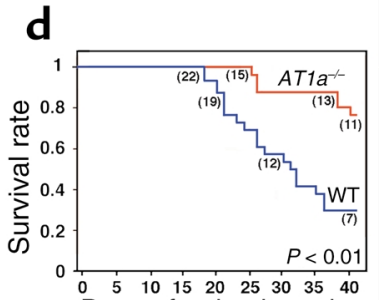

Days after implantation
X-ray microangiography. We performed postmortem tumor microangiography on day 21 after B16-F1 melanoma cell implantation. We found that the formation of tumor-feeding vessels visible with angiography was less potent in tumors engrafted in $A T 1 a^{-/-}$mice compared with those engrafted in WT mice (Figure 3, a and b).

Capillary density. We evaluated the capillary density by immunohistochemistry, since this is a measure of tumorassociated capillary angiogenesis. The density of CD31-positive capillary vessels inside tumors was significantly lower in $A T 1 a^{-/-}$mice (18 $\pm 3 /$ field) than in WT mice (37 $\pm 4 /$ field) (Figure 3, c, d, and i). The density of vWF-positive vessels in subcutaneous tissue surround-

\section{Figure 3}

Decreased vascular density in and around B16-F1 melanomas implanted in $A T 1 a^{-/-}$mice. Serial photo panels indicate representative $x$-ray microangiograms of melanomas grown in WT (a) or AT1 $a^{-1-}$ (b) mice, CD31-stained melanoma mass grown in WT $(\mathbf{c})$ or $A T 1 a^{-/-}$mice $(\mathbf{d})(\times 200)$, vWFstained subcutaneous tissues surrounding B16-F1 melanomas grown in WT (e) or $A T 1 a^{-/-}$mice (f) $(\times 200)$, and alkaline phosphatase-stained skeletal muscle tissues underlying B16-F1 melanomas grown in WT $(\mathbf{g})$ or $A T 1 a^{--}$mice $(\mathbf{h})(\times 400)$. Bars indicate $50 \mu \mathrm{m}$. The capillary densities of (i) tumors, (j) subcutaneous tissue surrounding tumors, and (k) skeletal muscles underlying tumors were all significantly smaller in $A T 1 a^{-/-}$ mice $(n=6)$ than in WT mice $(n=6)$. Values represent the average number of vessels at $\times 200 \pm$ the SE (by Student $t$ test).

\section{Figure 2}

Host-derived AT1a receptor is important for tumor growth. (a) A total of $10^{6}$ B16-F1 melanoma cells were implanted subcutaneously into WT $(n=11)$ and $A T 1 a^{-/-}(n=12)$ mice. Tumor volumes were significantly smaller in the $A T 1 a^{-/-}$group than in the WT group. (b) The Kaplan-Meier analysis shows the rate of survival was greater in the $A T 1 a^{-/-}$group than in the WT group. Numbers in parentheses indicate the number of animals surviving at each time point. (c) A total of $4 \times 10^{5}$ QRsP-11 fibrosarcoma cells were implanted subcutaneously into WT $(n=22)$ or AT1 $a^{-/-}(n=15)$ mice. Tumor volumes were significantly smaller in the $A T 1 a^{-/-}$group than in the WT group. (d) The Kaplan-Meier analysis shows the rate of survival was greater in the $A T 1 a^{-/}$group than in the WT group. Numbers in parentheses indicate the number of animals surviving at each time point.

ing tumors was also lower in $A T 1 a^{-/-}$mice ( $30 \pm 2 /$ field) than in WT mice (52 $\pm 8 /$ field) (Figure $3, \mathrm{e}, \mathrm{f}$, and $\mathrm{j}$ ). Likewise, the density of alkaline phosphatase-positive capillary endothelial cells within skeletal muscles underlying tumors was significantly lower in $A T 1 a^{-/-}$mice (26 $\pm 5 /$ field) than in WT mice (38 $\pm 5 /$ field) (Figure 3, $\mathrm{g}, \mathrm{h}$, and $\mathrm{k}$ ). Overall, we found that the tumor-associated capillary-density score was significantly lower in tissues obtained from $A T 1 a^{-/-}$mice compared with those obtained from WT mice.

AT1 a receptor expression. We examined tissue AT1a receptor expression. First, RT-PCR analysis in isolated
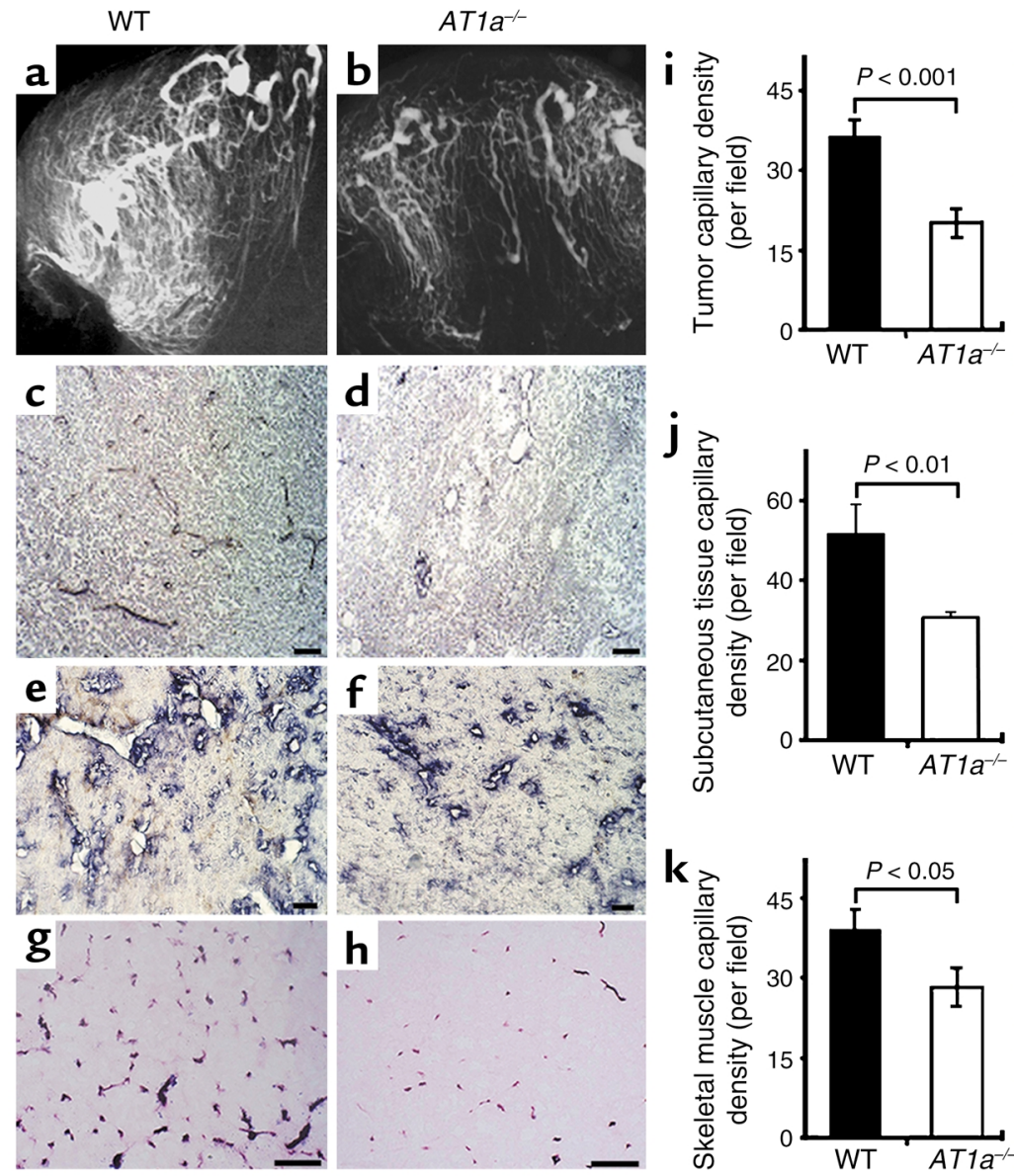
tissues revealed that cultured B16-F1 melanoma cells themselves expressed AT1 a receptor mRNA. In tumorengrafted WT mice, AT1a receptor mRNA was expressed in tumors and subcutaneous tissues surrounding tumors (Figure 4a). On the other hand, in tumor-engrafted $A T 1 a^{-/-}$mice, AT1a receptor mRNA was expressed only in tumor tissues that derived from implanted B16-F1 melanoma cells themselves. We found trace expression of AT1a receptor mRNA in subcutaneous tissues surrounding tumors, which presumably derived from B16-F1 melanoma cells invading from implanted tumors (Figure 4a). We then examined $\beta$-galactosidase mRNA expression by RT-PCR in tissues obtained from $A T 1 a^{-/-}$mice, because the $A T 1 a$ receptor gene was deleted and the $\beta$-galactosidase gene was inserted into the AT1a receptor gene locus (15). RT-PCR analysis revealed that $\beta$-galactosidase mRNA was expressed predominantly in subcutaneous tissues overlying tumors and was little expressed in tumors (Figure $4 \mathrm{~b})$. The intensity of the $\beta$-galactosidase mRNA expression was greater in subcutaneous tissues overlying tumors than in normal subcutaneous tissues. Accordingly, the host AT1a receptor expression as determined by $\beta$-galactosidase expression was enhanced in tissues surrounding tumors but not in normal subcutaneous tissues in $A T 1 a^{-/-}$mice.

Tissue inflammatory responses. From the results above, we see that the host AT1a receptor is mainly expressed in tissues surrounding tumors. Recent studies indicated that the RAS plays important proinflammatory roles in various disease models $(16,23,33,34)$, and inflammation is an important trigger for angiogenesis
$(26,35)$. We therefore examined inflammatory responses around tumor tissues isolated on postimplantation day 21. Standard H\&E staining revealed an infiltration of inflammatory leukocytes in WT mice (Figure 4c). We then stained tissue sections using the F4/80 mAb to detect macrophages, because TAMs are important triggers for tumor angiogenesis. The quantitative analysis revealed that the number of infiltrated $\mathrm{F} 4 / 80$-positive TAMs was significantly lower in $A T 1 a^{-/-}$mice than in WT mice (Figure 4c). Interestingly, immunohistochemical examination using anti- $\beta$-galactosidase $\mathrm{mAb}$ revealed that the major site of the expression of AT1a receptor was TAMs (Figure 4c).

Macrophages express angiogenic cytokine VEGF. TAMs release various angiogenic cytokines, including VEGF, that promote tumor neovascularization (24-27). To further examine the relationship between infiltrated TAMs and VEGF expression in tissues, we performed double-immunofluorescence staining for VEGF and the macrophage marker, F4/80. VEGF and F4/80 double-positive macrophages were predominantly located in subcutaneous tissues surrounding tumors (Figure 5a). The number of infiltrated VEGFpositive TAMs was less in $A T 1 a^{-/-}$mice than in WT mice (Figure $5 \mathrm{~b}$ ). ELISA of tissue homogenates revealed that tissue levels of VEGF and MCP- 1 proteins were significantly lower in $A T 1 a^{-/-}$mice than in WT mice (Figure 5b); however, the levels of VEGF protein in homogenized tumor masses standardized with total protein concentration were not significantly different between the two groups $(21 \pm 1.9$ in WT versus $24 \pm 1.3 \mathrm{pg} / \mathrm{mg}$ protein).

\section{Figure 4}

Host AT1a receptor is expressed on tumor-associated macrophages. (a) RT-PCR analysis for AT1a mRNA shows cultured B16-F1 melanoma cells, and implanted tumor tissues express AT1a mRNA. Subcutaneous tissues surrounding tumors expressed AT1a mRNA in WT mice but only slightly in $A T 1 a^{-/-}$mice. (b) RT-PCR analysis for $\beta$-galactosidase ( $\beta$-gal) mRNA in $A T 1 a^{-/-}$mice shows subcutaneous tissues surrounding tumors express $\beta$-galactosidase (equivalent expression site of host AT1 a receptor). $\beta$-Galactosidase mRNA is little expressed in tumors, indicating the absence of the host AT1 a receptor within tumor tissues. (c) Subcutaneous tissues isolated from a remote normal skin and tumor-implanted site were stained with an FITC-conjugated anti- $\beta$-galactosidase $\mathrm{mAb}$ (representing host AT1a receptor) (FITC- $\beta$-gal) and phycoerythrin-conjugated anti-macrophage mAb (PEmacrophage). Panels indicate that macrophages located around tumors (TAMs) express $\beta$-galactosidase (host AT1a receptor). Bars indicate $100 \mu \mathrm{m}$. T, tumor. $\mathbf{a}$
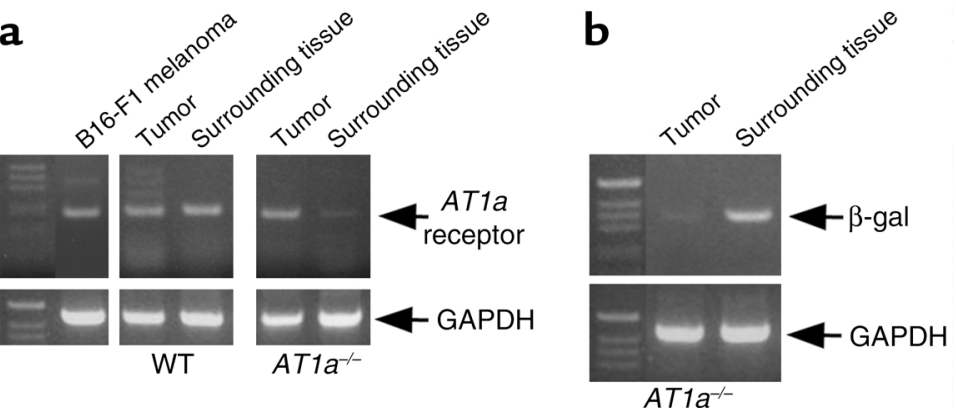

c
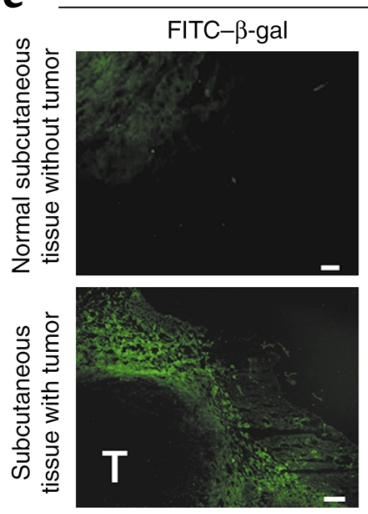

AT1a $^{-/-}$mice
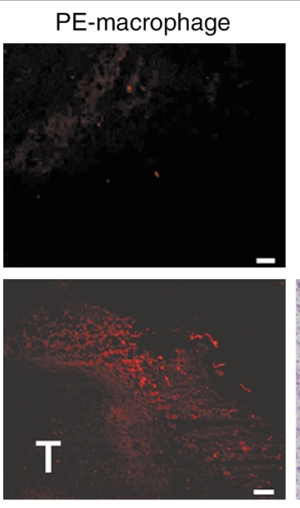

$H \& E$

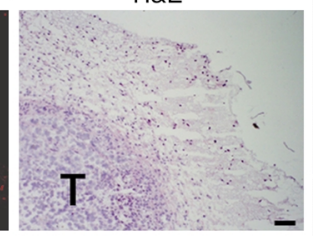



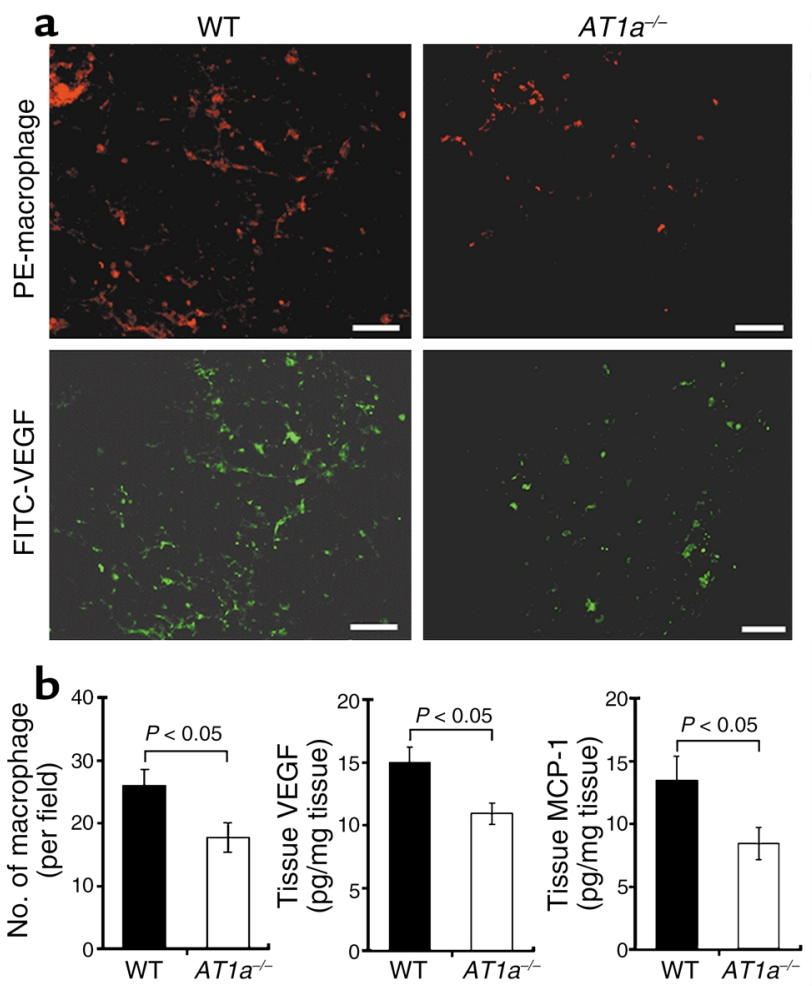

Effects of TCV-116 on melanoma angiogenesis and growth. Because subcutaneous melanoma-induced angiogenesis and growth were reduced in $A T 1 a^{-/-}$ mice, we evaluated the effects of a selective AT1 receptor blocker on tumor angiogenesis in WT mice in vivo. Treatment with TCV-116, a selective AT1 receptor blocker, inhibited melanoma growth and angiogenesis assessed by microangiography (Figure 6 , $\mathrm{a}$ and $\mathrm{b}$ ). Thus, pharmacological blockade with AT1 receptor also inhibits melanoma-induced angiogenesis and growth, which mimicked tumor growth suppression observed in $A T 1 a^{-/-}$mice.

\section{Discussion}

Although the RAS is an important system in regulating vascular homeostasis, the precise roles of the RAS and the ATII-AT1 receptor pathway in angiogenesis, especially in tumor-related angiogenesis, are unclear. To elucidate this issue, we took advantage of using genetically modified $A T 1 a^{-/-}$mice that we had generated recently $(15,16)$. In the present study,we demonstrate, we believe for the first time, that the host ATII-AT1 receptor pathway plays an important role in tumorrelated angiogenesis and growth in vivo. Moreover, these phenomena in $A T 1 a^{-/-}$mice were at least in part mediated by reduced TAM infiltration, an important determinant of tumor angiogenesis.

Tumor growth requires the maintenance and expansion of a vascular network $(3,4)$. In fact, various angiogenesis inhibitors have been shown to suppress tumor growth and to induce tumor dormancy $(28,36,37)$. In the present study, using two different tumor cell lines (B16-F1 melanoma and QRsP-11 fibrosarcoma cells),

\section{Figure 5}

TAMs express an angiogenic cytokine VEGF. (a) Macrophages were stained with a PE-conjugated anti-macrophage $m A b(F 4 / 80)$ in subcutaneous tissues surrounding tumors. Macrophages were costained with FITC-conjugated anti-VEGF mAb (FITC-VEGF). Bars indicate 50 $\mu \mathrm{m}$. (b) Macrophages were counted under fluorescence microscopy $(\times 200)$. The number of infiltrated macrophages was significantly lower in $A T 1 a^{-/-}$mice $(n=5)$ than in WT mice $(n=5)$. Tissue VEGF and MCP-1 protein levels were significantly lower in $A T 1 a^{-/-}$mice $(n=5)$ than in WT mice $(n=5)$.

we found that in vivo tumor growth was significantly inhibited in $A T 1 a^{-/}$mice compared with WT mice. Consistently, the survival rate of host animals after tumor implantation was significantly greater in $A T 1 a^{-/-}$ mice than in WT mice. Among these two cell lines, we focused on B16-F1 melanoma cells because melanoma growth depends greatly on angiogenesis (20, 25-27). Moreover, infiltration of monocytes/macrophages is critical for progression of melanomas toward an aggressive phenotype (38), and macrophage infiltration correlates with the degree of angiogenesis and disease stage (27). Therefore, the present melanoma implantation model is a useful system to use to analyze tumor angiogenesis, tumor growth, tumor-related macrophage infiltration, and host survival simultaneously.

We found that B16-F1 melanoma growth and angiogenesis were significantly reduced in $A T 1 a^{-/-}$mice. Moreover, in $A T 1 a^{-/-}$mice, the inhibitory efficacy of

a
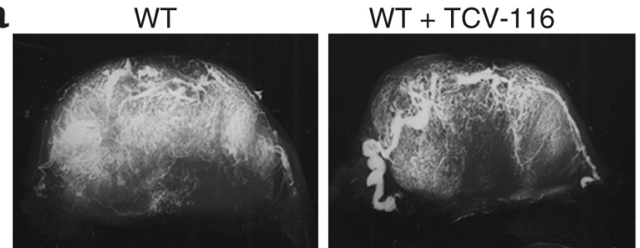

b

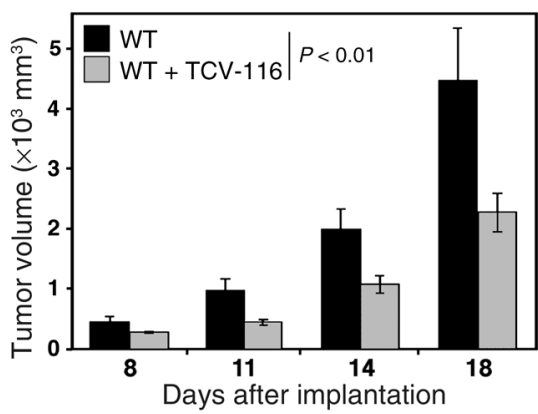

Figure 6

Suppression of tumor angiogenesis and growth in WT mice by treatment with TCV-116, a selective AT1 receptor blocker. (a) Representative $x$-ray microangiograms of melanomas grown in WT mice with (right) or without (left) TCV-116. (b) A total of $10^{6}$ B16-F1 melanoma cells were implanted subcutaneously into 33 WT mice with $(n=17)$ or without $(n=16)$ TCV-116 $(10 \mathrm{mg} / \mathrm{kg} /$ day $)$. Tumor volumes were calculated from tumor measurements scored at the indicated postimplantation day. The growth of B16-F1 melanoma was significantly reduced and delayed in WT mice treated with TCV-116 compared with control WT mice. 
TNP-470 on tumor growth was less potent as compared with that in WT mice. The latter finding suggests that the AT1a receptor deficiency almost sufficiently inhibited tumor angiogenesis, which may have limited further antiangiogenic (antitumor growth) efficacy of TNP-470. There may be several possible explanations for the reduced tumor angiogenesis in $A T 1 a^{-/-}$mice. Recent studies show that ATII acts as a proinflammatory molecule for immune-privileged tissues and cells $(33,34)$. In fact, macrophages express AT1 receptor intensively, and ATII enhances macrophage inflammatory functions through the AT1 receptor (22). Studies also suggest that infiltration of TAMs plays a pivotal role in tumor angiogenesis, enabling tumor cells to survive and proliferate (25), because macrophages can release various angiogenic growth factors, including VEGF, PDGF, IGF-1, bFGF, GM-CSF, IL-1, IL-6, IL-8, and TNF- $\alpha$, which stimulate tumor growth. VEGF is one of the most prominent angiogenic cytokines among those factors and is released from infiltrated TAMs $(23,25)$. We reported recently that macrophage infiltration, VEGF release from macrophages, and angiogenesis were significantly reduced in $A T 1 a^{-/-}$mice compared with WT mice in ischemic tissues (23). It is therefore conceivable that melanoma-associated macrophage infiltration and their cytokine release, especially VEGF, might be impaired, and thereby melanoma growth was retarded in $A T 1 a^{-/-}$mice in the present study.

To further address these issues, we examined inflammatory response and VEGF protein expression in tumor-associated tissues. First, we found that the number of infiltrated macrophages was significantly lower in $A T 1 a^{-/-}$mice than in WT mice in subcutaneous tissues surrounding tumors (approximately $3,000 \mu \mathrm{m}$ from tumor margin). Second, infiltrated macrophages intensively expressed VEGF protein, and the level of VEGF protein was significantly lower in $A T 1 a^{-/-}$mice than in WT mice in tissues surrounding tumors. Third, RT-PCR analysis revealed that host AT1a receptor expression (AT1a mRNA in WT mice and $\beta$-galactosidase mRNA in $A T 1 a^{-/-}$mice) was located mainly in tissues surrounding tumors, and immunohistochemical analysis in $A T 1 a^{-/-}$mice revealed that $\beta$-galactosidase protein was predominantly expressed on infiltrated TAMs. Thus, our findings suggest that the host AT1a receptor is preferentially expressed on TAMs, which release VEGF, and therefore the ATIIAT1a receptor pathway may play important roles in promoting tumor angiogenesis and growth in a TAMand VEGF-dependent manner. These are previously unknown important functions of the ATII-AT1 receptor pathway in tumor biology.

There are some limitations in the present study. First, we examined only two tumor types in one mouse strain (i.e., B16-F1 melanoma cells and QRsP-11 fibrosarcoma cells in C57BL/6 mice). Other tumor types combined with other experimental conditions should be analyzed. In this regard, two recent reports show that pharmacological blockade of AT1 receptor also reduced tumor angiogenesis, growth, and metastasis $(39,40)$, further supporting our findings. Second, the AT1 receptor is expressed on not only macrophages but also endothelial cells and VSMCs. Indeed, ATII has been shown to stimulate production of VEGF from VSMCs, and ATII directly enhances endothelial capillary network formation $(41,42)$. Thus, these mechanisms should also be involved in the reduced angiogenesis in $A T 1 a^{-/-}$mice. Third, we used WT mice treated with a relatively high dose of TCV-116. Although the present regimen of TCV-116 administration does not elicit any cytotoxic actions in rodents $(43,44)$, our data may not be directly extrapolated to humans receiving clinical doses of TCV-116. We will need to analyze the doserelated effects of AT1 receptor blockers on tumor angiogenesis in vivo in the future. Finally, there is a possibility that melanoma itself releases VEGF protein that induces angiogenesis. Although the VEGF levels within tumor masses standardized with total protein were similar to each other between the two groups, the size of tumor mass was much smaller in $A T 1 a^{-/-}$mice than in WT mice. Therefore, the overall release of VEGF protein from tumor mass could be still smaller in $\mathrm{ATI}^{-/-}$ mice than in WT mice.

In summary, our findings suggest that the host ATIIAT1 receptor pathway plays a significant role in tumor angiogenesis and growth in vivo. The mechanism is at least in part related to the ATII-mediated inflammatory TAM infiltration. These are newly discovered functions of the ATII-AT1 receptor pathway. Our study not only provides a novel insight into the pathogenesis of tumor angiogenesis, but also hints at a possible new therapeutic strategy against tumors.

\section{Acknowledgments}

This study was supported by Research Grants 12136208 and 14370235 and a Scientific Frontier grant from the Ministry of Education, Science and Culture, the Tokyo Biochemical Research Society, the Japan Heart Foundation, the Terumo Research Foundation, and grants from the Ministry of Health and Welfare (to T. Murohara). We thank K. Kimura, K. Moriyama, and M. Aoki for technical assistance, and Takeda Chemical Industries Inc. (Osaka, Japan) for TCV-116 and TNP-470.

\footnotetext{
1. Brunner, H.R., et al. 1972. Essential hypertension: rennin and aldosterone, heart attack and stroke. N. Engl. J. Med. 286:441-449.

2. Lever, A.F., et al. 1998. Do inhibitors of angiotensin-I-converting enzyme protect against risk of cancer? Lancet. 352:179-184.

3. Folkman, J. 1995. Angiogenesis in cancer, vascular, rheumatoid and other disease. Nat. Med. 1:27-31.

4. Hanahan, D., and Folkman, J. 1996. Patterns and emerging mechanisms of the angiogenic switch during tumorigenesis. Cell. 86:353-364.

5. Volpert, O.V., et al. 1996. Captopril inhibits angiogenesis and slows the growth of experimental tumors in rats. J. Clin. Invest. 98:671-679.

6. Fabre, J.-E., Rivard, A., Magner, M., Silver, M., and Isner, J.M. 1999. Tissue inhibition of angiotensin-converting enzyme activity stimulates angiogenesis in vivo. Circulation. 99:3043-3049.

7. Takeshita, S., et al. 2001. Angiotensin-converting enzyme inhibition improves defective angiogenesis in the ischemic limb of spontaneously hypertensive rats. Cardiovasc. Res. 52:314-320.

8. Warren, J.B., and Loi, R.K. 1995. Captopril increases skin microvascular
} 
blood flow secondary to bradykinin, nitric oxide, and prostaglandins. FASEB J. 9:411-418.

9. Murohara, T., et al. 1998. Nitric oxide synthase modulates angiogenesis in response to tissue ischemia. J. Clin. Invest. 101:2567-2578.

10. Muramatsu, M., Katada, J., Hayashi, I., and Majima, M. 2000. Chymase as a proangiogenic factor. A possible involvement of chymaseangiotensin-dependent pathway in the hamster sponge angiogenesis model. J. Biol. Chem. 275:5545-5552.

11. Timmermans, P.B., et al. 1993. Angiotensin II receptors and angiotensin II receptor antagonists. Pharmacol. Rev. 45:205-251.

12. Burnier, M. 2001. Angiotensin II type 1 receptor blockers. Circulation. 103:904-912.

13. Viswanathan, M., Stromberg, C., Seltzer, A., and Saavedra, J.M. 1992. Balloon angioplasty enhances the expression of angiotensin II AT1 receptors in neointima of rat aorta. J. Clin. Invest. 90:1707-1712.

14. Harada, K., Komuro, I., Sugaya, T., Murakami, K., and Yazaki, Y. 1999. Vascular injury causes neointimal formation in angiotensin II type 1a receptor knockout mice. Circ. Res. 84:179-185.

15. Sugaya, T., et al. 1995. Angiotensin II type 1a receptor-deficient mice with hypotension and hyperreninemia. J. Biol. Chem. 270:18719-18722.

16. Hisada, Y., et al. 1999. Angiotensin II plays a pathogenic role in immunemediated renal injury in mice J. Clin. Invest. 103:627-635.

17. Williams, C.S., Tsujii, M., Reese, J., Dey, S.K., and DuBois, R.N. 2000 Host cyclooxygenase-2 modulates carcinoma growth. J. Clin. Invest. 105:1589-1594.

18. Habelhah, H., et al. 1998. Polysaccharide K induces Mn superoxide dismutase (Mn-SOD) in tumor tissues and inhibits malignant progression of QR-32 tumor cells: possible roles of interferon alpha, tumor necrosis factor alpha and transforming growth factor beta in Mn-SOD induction by polysaccharide K. Cancer Immunol. Immunother. 46:338-344.

19. Wang, J., et al. 1995. Demonstration that mutation of the type II transforming growth factor beta receptor inactivates its tumor suppressor activity in replication error-positive colon carcinoma cells. J. Biol. Chem. 270:22044-22049.

20. Gorrin-Rivas, M.J., et al. 2000. Mouse macrophage metalloelastase gene transfer into a murine melanoma suppresses primary tumor growth by halting angiogenesis. Clin. Cancer Res. 6:1647-1654.

21.Ziada, A.M., Hudlicka, O., Tyler, K.R., and Wright, A.J. 1984. The effect of long-term vasodilation on capillary growth and performance in rabbit heart and skeletal muscle. Cardiovasc. Res. 18:724-732.

22. Okamura, A., et al. 1999. Upregulation of renin-angiotensin system during differentiation of monocytes to macrophages. J. Hypertens. 17:537-545

23. Sasaki, K., et al. 2002. Evidence for the importance of angiotensin II type 1 receptor in ischemia-induced angiogenesis. J. Clin. Invest. 109:603-611. doi:10.1172/JCI200213055.

24. Leibovich, S.J., et al. 1987. Macrophage-induced angiogenesis is mediated by tumour necrosis factor- $\alpha$. Nature. 329:630-632.

25. Sunderkoetter, C., Steinbrink, K., Goebeler, M., Bhardwaj, R., and Sorg, C. 1994. Macrophages and angiogenesis. J. Leukoc. Biol. 55:410-422.

26. Gutman, M., et al. 1994. Leukocyte-induced angiogenesis and subcutaneous growth of B16 melanoma. Cancer Biother. 9:163-170.
27. Torisu, H., et al. 2000. Macrophage infiltration correlates with tumor stage and angiogenesis in human malignant melanoma: possible involvement of TNF-alpha and IL-7-alpha. Int. J. Cancer. 85:182-188.

28. Ingber, D., et al. 1990. Synthetic analogues of fumagillin that inhibit angiogenesis and suppress tumour growth. Nature. 348:555-557.

29. Kato, T., Sato, K., Kakinuma, H., and Matsuda, Y. 1994. Enhanced suppression of tumor growth by combination of angiogenesis inhibitor O-(chloroacetyl-carbamoyl)fumagillol (TNP-470) and cytotoxic agents in mice. Cancer Res. 54:5143-5147.

30. Yoshiyama, M., et al. 1994. Cardioprotective effect of the angiotensin II type 1 receptor antagonist TCV-116 on ischemia-reperfusion injury. Am. Heart J. 128:1-6.

31. Kojima, M., et al. 1994. Angiotensin II receptor antagonist TCV-116 induces regression of hypertensive left ventricular hypertrophy in vivo and inhibits the intracellular signaling pathway of stretch-mediated cardiomyocyte hypertrophy in vitro. Circulation. 89:2204-2211.

32. Kaplan, E.L., and Meier, P. 1958. Nonparametric estimation from incomplete observations. J. Am. Stat. Assoc. 53:457-481.

33. Usui, M., et al. 2000. Important role of local angiotensin II activity mediated via type 1 receptor in the pathogenesis of cardiovascular inflammatory changes induced by chronic blockade of nitric oxide synthesis in rats. Circulation. 101:305-310.

34. Nataraj, C., et al. 1999. Angiotensin II regulates cellular immune responses through a calcineurin-dependent pathway. J. Clin. Invest. 104:1693-1701.

35. Arras, M., et al. 1998. Monocyte activation in angiogenesis and collateral growth in the rabbit hindlimb. J. Clin. Invest. 101:40-50.

36. O'Reilly, M.S., et al. 1997. Endostatin: an endogenous inhibitor of angiogenesis and tumor growth. Cell. 88:277-285.

37. O'Reilly, M.S., Holmgren, L., Chen, C., and Folkman, J. 1996. Angiostatin induces and sustains dormancy of human primary tumors in mice. Nat. Med. 2:689-692.

38. Brocker, E.B., Zwadlo, G., Suter, L., Brune, M., and Sorg, C. 1987. Infiltration of primary and metastatic melanomas with macrophages of the 25F9-positive phenotype. Cancer Immunol. Immunother. 25:81-86.

39. Fujita, M., Hayashi, I., Yamashina, S., Itoman, M., and Majima, M. 2002. Blockade of angiotensin AT1a receptor signaling reduces tumor growth, angiogenesis, and metastasis. Biochem. Biophys. Res. Commun. 294:441-447.

40. Miyajima, A., et al. 2002. Angiotensin II type I antagonist prevents pulmonary metastasis of murine renal cancer by inhibition tumor angiogenesis. Cancer Res. 62:4176-4179.

41. Otani, A., Takagi, H., Suzuma, K., and Honda, Y. 1998. Angiotensin II potentiates vascular endothelial growth factor-induced angiogenic activity in retinal microcapillary endothelial cells. Circ. Res. 82:619-628.

42. Williams, B., Baker, A.Q., Gallacher, B., and Lodwick, D. 1995. Angiotensin II increases vascular permeability factor gene expression by human vascular smooth muscle cells. Hypertension. 25:913-917.

43. Sato, K., et al. 1996. Four-week oral toxicity study of TCV-116 in rats. Japanese Pharmacology and Therapeutics. 25:11-28.

44. Nishida, N., et al. 1996. Twenty-six week oral toxicity study of TCV-116 in rats. Japanese Pharmacology and Therapeutics. 24:49-68. 